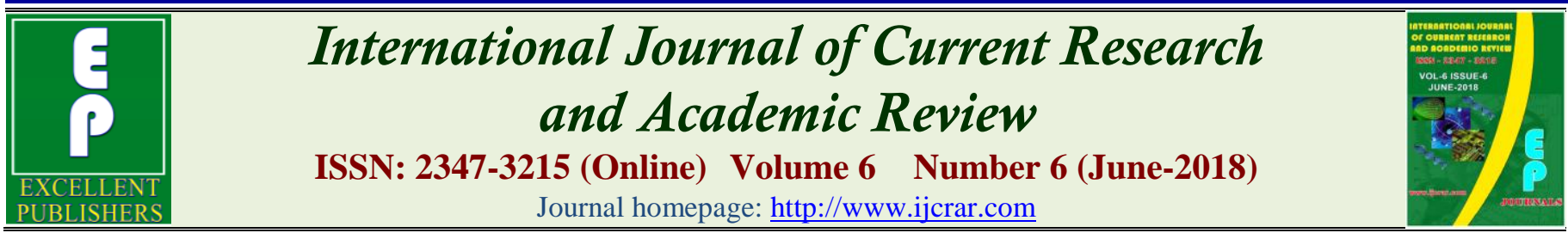

doi: https://doi.org/10.20546/ijcrar.2018.606.003

\title{
Berberine Binds in Silico to Anti-cancer Drug Target Enzyme Phosphoinositide 3- Kinase (Human PI3 K) with Affinity Comparable to Known Inhibitors of the Enzyme
}

\author{
K.S.V. Prabhu Rathnam ${ }^{1,2}$ and C. Damaodara Reddy ${ }^{1}$ * \\ ${ }^{1}$ Sugen Life Sciences Pvt.Ltd. 4/86, S.V.Nagar Perumallapalli, Tirupathi-517502, India \\ ${ }^{2}$ S.V.University, Tirupathi -517502 , India
}

*Corresponding author

\begin{abstract}
Phosphoinositide 3-kinase (PI3K) is an intracellular enzyme functioning as an inositol lipid kinase and is activated by several upstream growth factor receptors. PI3 K controls diverse cellular responses including but not limited to cell proliferation, survival, etc. Several recent reports have undoubtedly proved that PI $3 \mathrm{~K}$ is a druggable target and several research groups and pharmaceutical companies are developing inhibitors. In the present study, we present the results of Insilco docking analysis of a phytocompound Berberine and were compared with several known commercial PI3 K inhibitors. Our results show that Berberine docks efficiently with the active site of PI3 K and forms stable bonds with active site amino acid residues and essentially stalls enzyme activity. Our results also show that the bonding energies of these interactions are far more superior to the commercial PI3 K inhibitors and probably advocates as a probable PI3 K inhibitor.
\end{abstract}

\section{Article Info}

Accepted: 28May 2018

Available Online: 20 June 2018

\section{Keywords}

Berberine PI3K, Breast cancer, Triple negative, Epirubicin.

\section{Introduction}

Triple-negative breast cancers are characterized by lack of expression of both estrogen receptor (ER) and progesterone receptor (PR), as well as absence of human epidermal growth factor 2 (HER2) up regulation and accounts to $10-17 \%$ of all breast carcinomas (Shoemaker et al.(2018)). TNBCs are associated with an overall poor prognosis as exemplified by a higher rate of early recurrence and distant metastasis to brain and lungs compared to other breast cancer subtypes. The treatment options for TNBCs are limited. Although, early TNBC can be sensitive to standard chemotherapy (anthracyclines, taxanes, ixabepilone, and platinum agents), traditional hormone therapies and targeted agents such as trastuzumab are not effective in this phenotype of cancer (Dent et al.(2007)). Recent studies have indicated that the accumulations of defects in cellular regulation mechanisms are underlying the cause for resistance to conventional chemotherapy accounting for $90 \%$ of drug failures in TNBC's (Foulkes et al., (2010)). Therefore, to enhance treatment efficacy, targeted therapies to specific signaling molecules overexpressed and implicated in the pathogenesis of TNBCs holds promise in treatment. 
Bioactive compounds from dietary phytochemicals are well studied for their chemopreventive and chemotherapeutic effects. Investigations of several bioactive compounds revealed their ability to function as multi-targeting agents to antagonize dysregulated targets in cellular signaling pathways to exert their antineoplastic activities (Saldanha et al., 2012). Altering genes that affect these pathways can serve as suitable tools to decrease tumor mass and also allow for tumor regression. Various studies have shown that these molecules can induce apoptosis, inhibit cellular proliferation, affect angiogenesis, and affect cancer metabolism in various cancers, all of which are hindrances to tumor growth. Thus an effective nutraceutical is one that will have a low nontoxic dose to create the magnitude of death inducing changes in the tumor dynamics. Phytochemical modulate an array of signaling pathways, as well as individual molecular targets. Available literature reveals that natural anticancer agents have been shown to touch upon virtually every single molecular target (Sun et al., 2002). A few major signaling pathways/targets affected by nutraceutical or phytocompound that have been characterized include but not limited to: EGFR family of receptors, Ras/Raf signaling, PI3K/Akt/mTOR pathway, Notch family, Wnt/ $\beta$-catenin signaling, MAPK/ERK pathway, among others (Torres et al, 2012 and Singh et al, 2011).

Berberine is a benzylisoquinoline alkaloid extracted from the roots, rhizomes, stem of several plants such as Berberis vulgaris-Barberry, Berberis aristata. Berberine has a molecular weight of $336.367 \mathrm{~g} / \mathrm{mol}$ and the molecular formula is $\mathrm{C} 20 \mathrm{H} 18 \mathrm{NO} 4$. The hydrogen bond donor count is 0 and acceptor count is 4 . It is a solid substance and has a melting point of $145^{\circ} \mathrm{C}$. The XlogP value is 3.6. It is slowly soluble in water.

Berberine is used as potent anti-diabetic drug which decreases the blood glucose level by various mechanisms such as decreasing insulin resistance, decreasing gluconeogenesis and increasing glycolysis. The anticancer role of Berberine is gaining importance and few international groups are working on deciphering the mechanism of action (LMG Ortiz et al, 2014). It is on these lines, the present study was undertaken to show for the first time the molecular docking of Berberine with an important signaling node active in cancer cells, namely, Phosphatidyl linositide 3-kinases (PI3 K). It catalyses the transfer of terminal phosphate to the membrane bound lipid phosphatidyl inositol and triggers a signaling cascade resulting in activation of several downstream proteins involved in cell proliferation and differentiation (Fiona M. Foster et al, 2003). Several epithelial tumor types have been shown to have dysregulated PI3 K levels and have been directly implicated in malignant transformation process (J. E. Dancey,2004).

\section{Materials and Methods}

\section{Molecular docking}

Molecular docking was carried out using MOE (Molecular Operating Environment) docking software tool (MOE 2011.10). The 3D structure of Berberine, Epirubicin, and known PI3-K inhibitors such as BKM120, CH5132799, GDC-0941, GDC0980, GSK 2126458 and LYS294002 was retrieved from PubChem (ID 2353) and its geometry was optimized in MOE working environment. The protein PI3 kinase structure was loaded into MOE software removing water molecules, hetero atoms and polar hydrogens were added. The structures were protonated at a temperature of $300 \mathrm{~K}, \mathrm{pH}$ 7 and the salt concentration was kept at $0.1 \mathrm{M}$. Generalized born implicit solvating environment was enabled with a dielectric constant of 1 and Vanderwaal's forces were enabled at a cut off value of $10 \AA$. Energy minimization was carried out in OPLS (Optimize Potential for Liquid Simulations) force field at a gradient of 0.05 to calculate the atomic coordinates of the protein that are local minima of molecular energy function and also to determine low energy conformations and to proceed for molecular dynamics simulations. The simulations were carried out in the same force field and NVT statistical ensemble was used where the temperature is held fixed to generate the trajectories. The most accurate Nose-Poincare-Anderson algorithm was enabled to solve the equation of motion during simulations. The initial temperature was set at $30 \mathrm{~K}$ and increased to a run time temperature of $300 \mathrm{~K}$ and the simulations were carried out for a total period of 10 nano seconds and these stabilized conformations generated at the end of the simulations were used for molecular docking process.

Individual dockings were performed for protein PI3 kinase with Berberine, Epirubicin, BKM-120, CH5132799, GDC-0941, GDC0980, GSK 2126458 and LYS294002 to find out the binding modes and affinity variations. These poses were generated by superposition of ligand atom triplets and triplets of receptor site points using alpha triangle docking placement methodology. The docked conformers are ranked by London $\mathrm{dG}$ scoring function to estimate the free energy of binding of 
the ligand from a given pose. The conformations thus were refined and rescored in the same force filed to remove the duplicate conformations (Ahmed Jeri et al., (2015)). At the end of docking process the pose with least score was chosen from the total conformations and in each docking process the binding orientations of Berberine and Epirubicin was studied in the binding sites of protein PI3-K (J. E. Dance (2004)).

\section{Results and Discussions}

The interaction pattern of the phytocompound Berberine with the cellular protein target namely PI3 Kinase suggest an important role in execution of its pharmacological effects. Consistent with our in-vitro findings, we were able to see good protein - ligand interaction with the cellular target PI3 Kinase, involved in the mechanism of action of Berberine.

To substantiate the findings for the in vitro studies, docking studies were performed and the target protein selected was the intra cellular signaling kinase, Phosphatidyl inositide 3- Kinase (PI3-K). Docking was performed with the substrate binding site of the kinase and the docking energies, bond formation were all recorded. In the present study, Berberine and Epirubicin were tested in the in vitro studies, nevertheless, docking was carried out with 6 other additional small molecule compounds, listed as PI3-K inhibitors. The 6 small molecules listed as PI3 Kinase inhibitors were chosen from Pubchem, BKM-120, CH5132799, GDC-0941, GDC0980, GSK 2126458 and LYS294002. Results of this docking study show among all the compounds that were docked with PI3-K, Berberine showed very promising results with a docking score of -10.5966 $\mathrm{kcal} / \mathrm{mol}$, while the next best was Epirubicin, which had a score of $-10.8622 \mathrm{kcal} / \mathrm{mol}$. On comparison the docking scores for the other small molecules were not significant. The results of the molecular docking studies, in terms of bond formation, energy are summarized in Table. 1. The 2-D docking poses of Berberine with the substrate binding site of PI3-Kinase and the interacting amino acid residues are shown in Figure-1. The 3-D docking poses of Berberine and Epirubicin is shown in Figure 2. The 2-D and 3-D docking poses of the other 6 small molecules are shown in Figures- 3 and 4 respectively.

Table.1 In Silico Docking scores of Berberine, Epirubicin and Selective Inhibitors with PI3Kinase

\begin{tabular}{|c|c|c|c|c|c|c|}
\hline S. No. & Drug & Target & Docking score & $\begin{array}{l}\text { No. of H- } \\
\text { Bonds }\end{array}$ & \begin{tabular}{|l|} 
Interacting \\
residues
\end{tabular} & $\begin{array}{l}\text { H-bond } \\
\text { length }(\AA)\end{array}$ \\
\hline \multirow{4}{*}{1} & \multirow{4}{*}{ Berberine } & \multirow{4}{*}{ PI3 kinase } & \multirow{4}{*}{-10.5966} & \multirow{4}{*}{4} & Ser50 & 2.3 \\
\hline & & & & & Ser50 & 2.5 \\
\hline & & & & & \begin{tabular}{|l} 
Lys71 \\
\end{tabular} & 2.8 \\
\hline & & & & & Ala49 & 3.0 \\
\hline \multirow{3}{*}{2} & \multirow{3}{*}{ Epirubicin } & \multirow{3}{*}{ PI3 kinase } & \multirow{3}{*}{-10.8622} & \multirow{3}{*}{3} & Lys63 & 3.0 \\
\hline & & & & & Asp41 & 2.4 \\
\hline & & & & & Asp41 & 2.6 \\
\hline 3 & BKM-120 & PI3 kinase & -10.1743 & - & - & - \\
\hline 4 & CH5132799 & PI3 kinase & -9.8694 & 1 & Arg47 & 2.7 \\
\hline 5 & GDC-0941 & PI3 kinase & -10.4513 & 1 & Asn33 & 2.2 \\
\hline \multirow{2}{*}{6} & \multirow{2}{*}{ GDC0980 } & \multirow{2}{*}{ PI3 kinase } & \multirow{2}{*}{-10.5462} & \multirow{2}{*}{2} & Asn67 & 2.5 \\
\hline & & & & & Asn67 & 2.8 \\
\hline 7 & GSK 2126458 & PI3 kinase & -10.0443 & - & - & - \\
\hline 8 & LYS294002 & PI3 kinase & -9.6507 & - & - & - \\
\hline
\end{tabular}


Figure.1 In Silico Docking of Berberine, Epirubicin - 2D Images of Docking with target PI3 Kinase



Figure.2 In Silico Docking of Berberine, Epirubicin - 3D Images of Docking with target PI3 Kinase

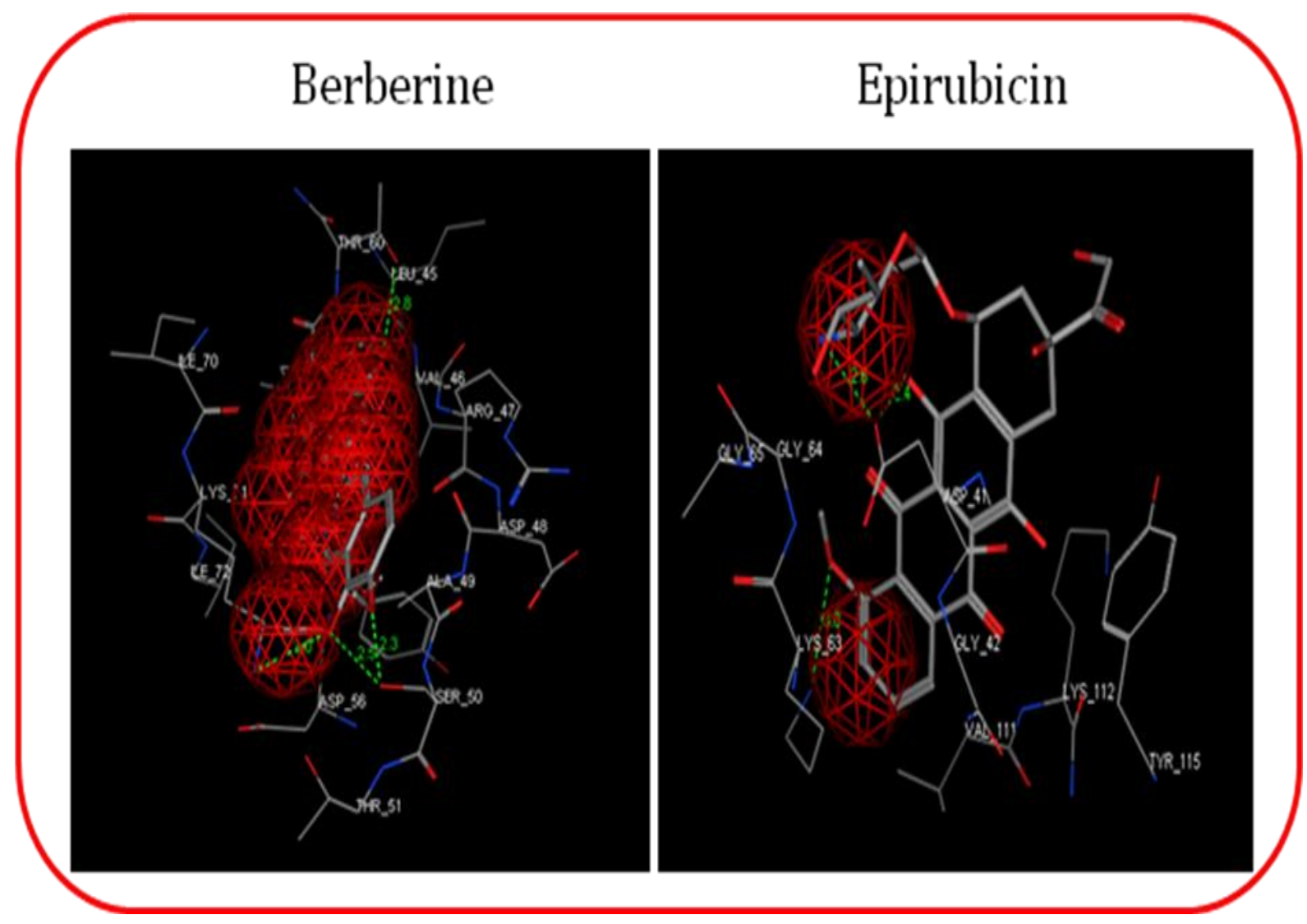


Figure.3 In Silico Docking of Selective Small molecules- 2D Images of Docking with target PI3 Kinase

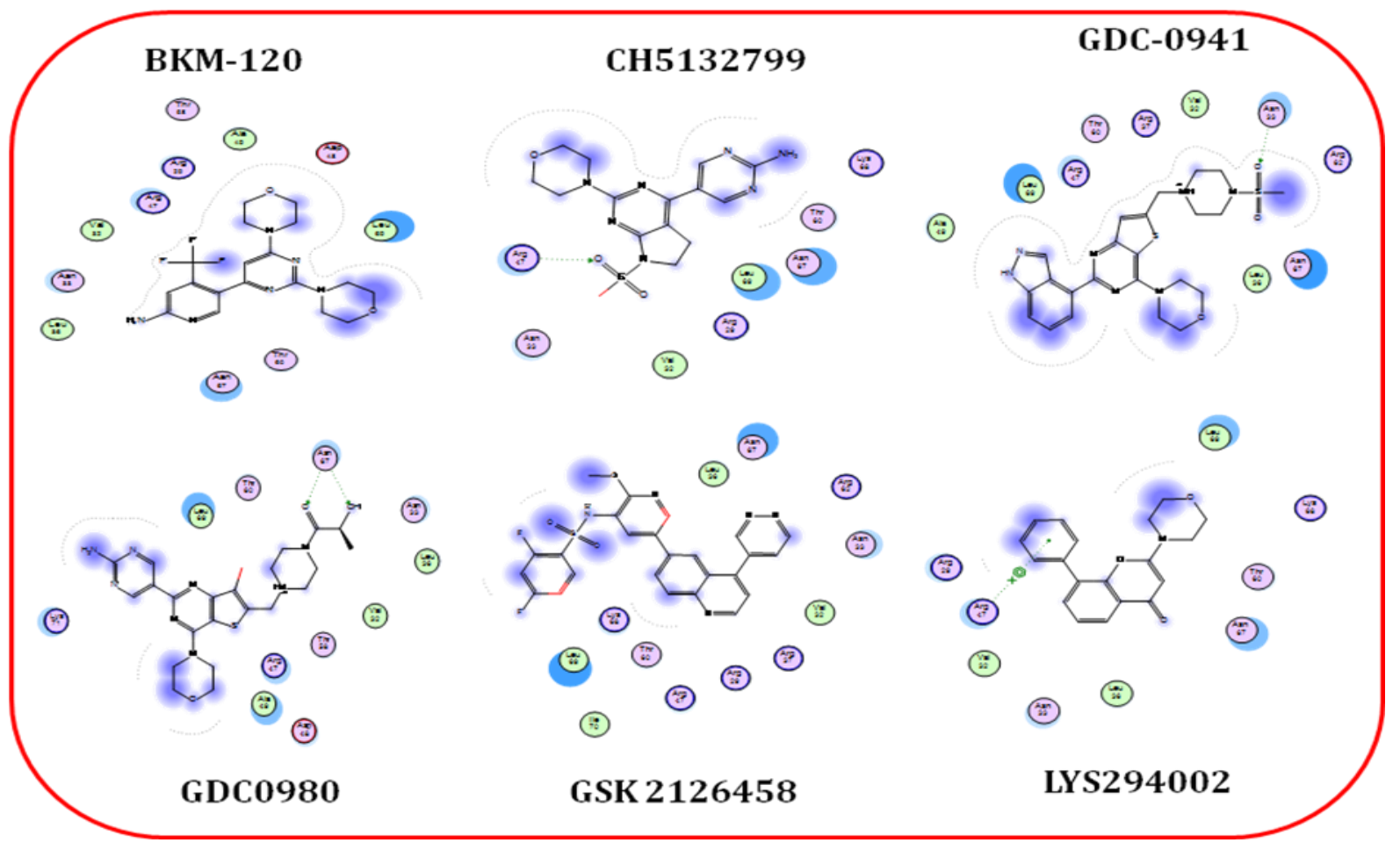

Figure.4 In Silico Docking of Selective Small Molecule Inhibitors - 3D Images of Docking with target PI3 Kinase

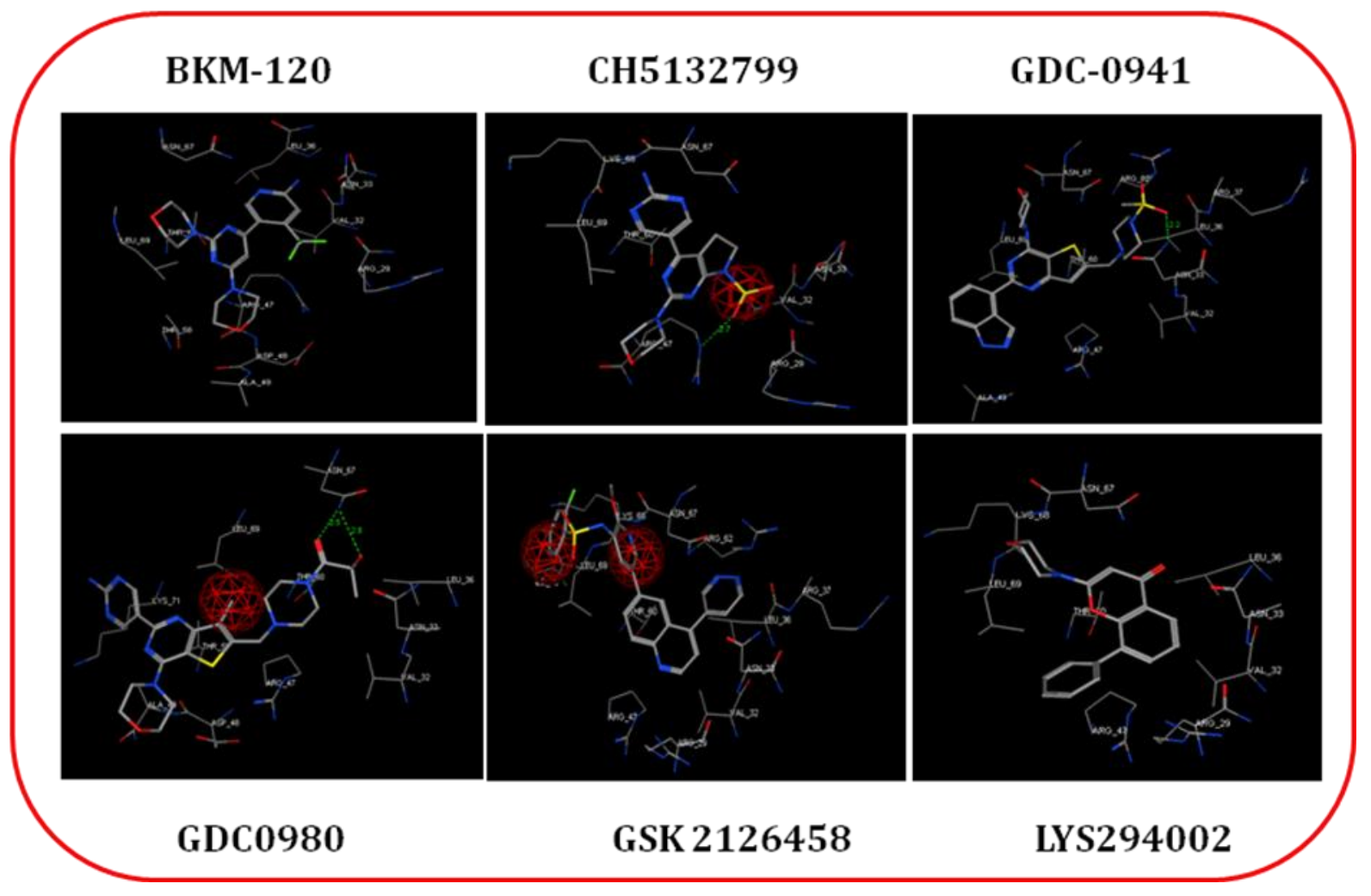


Further in depth analysis of the docking study for Berberine with the target kinase PI3-K showed that it interacts with important amino acid residues namely, Ser50, Ser 50, Lys71 and Ala 49 forming a total of four hydrogen bonds. The N-2 atom of the compound berberine formed a strong hydrogen bond interaction with the $\mathrm{NH}$ group of Ser50 and the distance was computed to be $2.3 \AA$. Furthermore, the N-2 atom of the compound berberine formed a strong hydrogen bond interaction with the $\mathrm{NH}$ group of Lys71 (distance $=2.5$ $\AA$ ). Finally, the N-2 atom of the compound berberine formed a strong hydrogen bond interaction with the with the NH group of Ala49 (distance=3.0 $\AA$ ).

Molecular docking studies of the substrate binding site of PI3-Kinase with Epirubicin revealed that it interacts with Lys63 and Asp41 forming a total of three hydrogen bonds. The N-2 atom of the compound epirubicin formed a strong hydrogen bond interaction with the with the $\mathrm{NH}$ group of Lys63 (distance $=3.0 \AA$ ) and the $\mathrm{N}-2$ group of epirubicin formed a strong hydrogen bond interaction with the NH group of Asp41. The 2-D docking poses of Berberine and Epirubicin with the substrate binding site of PI3-Kinase and the interacting amino acid residues are shown in Figure-1.

Overall, the docking studies show that Berberine can efficiently interact with the substrate binding site of PI3Kinase and probably may influence the activity of the enzyme and thereby may influence the downstream signalling in breast cancer cells. The in silico data is in alignment with the in vitro studies and essentially corroborates the effective anti cancer activity of Berberine and Epirubicin alone and in combination on breast cancer cells.

\section{References}

Ahmed Jeri, Yahiya Hoban, B Vend Kumar, and Anil Biwa. Curcumin binds in silico to anti-cancer drug target enzyme MMP-3 (human stromelysin-1) with affinity comparable to two known inhibitors of the enzyme. Misinformation. 2015; 11(8) 387392.

Dent R, Trudeau M, Pritchard KI, Hanna WM, Kahn HK, Sawka CA, Lickley LA, Rawlinson E, Sun P, Narod SA. Triple-negative breast cancer: clinical features and patterns of recurrence. Clin Cancer Res. 2007 Aug 1;13(15 Pt 1):4429-34.

Fiona M. Foster, Colin J. Traer, Siemon M. Abraham, Michael J. Fry. The Phosphoinositide (PI) 3-kinase family. Journal of Cell Science 2003. 116: 3037 3040.

Foulkes WD, Smith IE, Reis-Filho JS. Triple-negative breast cancer. N Engl JMed. 2010 Nov 11; 363(20):1938-48.

Dance, J. E. Molecular targeting: PI3 kinase pathway. Annals of Oncology2004, 15 (Supplement 4): iv233 - iv239.

LMG Ortiz, P Lombardi, M Tillhon, AI Scovazzi (2014) Berberine: an epiphany against cancer. Molecules. 19(8), 12349-12367.

Pub hem ID: 2353. URL:https://pubchem.ncbi.nlm.nih.gov/compound /2353\#section=Top

Saldanha SN, Tollefsbol TO. The role of nutraceuticals in chemoprevention and chemotherapy and their clinical outcomes. J Oncol. 2012: 1-23.

Shoemaker ML, White MC, Wu M, Weir HK, Romieu I. Differences in breast cancer incidence among young women aged 20-49 years by stage and tumor characteristics, age, race, and ethnicity, 2004-2013. Breast Cancer Res Treat. 2018 Jun; 169(3):595-606.

Singh T, Vaid M, Katiyar N, Sharma S, Katiyar SK. Berberine, an isoquinoline alkaloid, inhibits melanoma cancer cell migration by reducing the expressions of cyclooxygenase-2, prostaglandin $\mathrm{E}_{2}$ and prostaglandin $\mathrm{E}_{2}$ receptors. Carcinogenesis. 2011 Jan; 32(1):86-92.

Sun, J.; Chu, Y.-F.; Wu, X.; Liu, R. H. (2002). Antioxidant and antiproliferative activities of common fruits. J. Agric. Food Chem, 50, 74497454.

Torres et al, 2012 Torres MP, Rachagani S, Purohit V, Pandey P, Joshi S, Moore ED, Johansson SL, Singh PK, Ganti AK, Batra SK. Graviola: a novel promising natural-derived drug that inhibits tumorogenicity and metastasis of pancreatic cancer cells in vitro and in vivo through altering cell metabolism. Cancer letters. 2012 Oct 1; 323(1):2940.

\section{How to cite this article:}

Prabhu Rathnam, K.S.V. and Damaodara Reddy, C. 2018. Berberine Binds in Silico to Anti-cancer Drug Target Enzyme Phosphoinositide 3- Kinase (Human PI3 K) with Affinity Comparable to Known Inhibitors of the Enzyme. Int.J.Curr.Res.Aca.Rev. 6(6), 16-21. doi: https://doi.org/10.20546/ijcrar.2018.606.003 